\title{
Estado de arte de la investigación sobre la incidencia de las estra- tegias didácticas en el proceso de enseñanza-aprendizaje
}

\section{State of the art of research on the incidence of didactic strategies in the teaching-learning process}

(C) UNAN-Managua

Recibido: 24 de febrero 2021

Aprobado: 24 de marzo 2021
Tania Yaoska Busto Lara Investigadora independiente taniyahosbustos@gmail.com https://orcid.org/0000-0002-8894-9303

\section{(9)}

\section{RESUMEN}

El presente artículo científico tiene como fin evidenciar los avances investigativos sobre las estrategias didácticas y su efectividad en el proceso de enseñanza-Aprendizaje. En él se hace énfasis en la metodología, correspondiente a las fases de investigación documental. Primeramente, se indagó en sitios web los trabajos realizados en torno a este tema, dichos estudios son actualizados en una fecha no menor de 5 años, 50 porciento nacional y 50 porciento internacional. También se explica los resultados, el cual contempla los aportes teóricos, es decir aquella fundamentación conceptual que explica las variables de investigación, tal como: estrategia didáctica y su importancia en el proceso de aprendizaje. De igual manera se muestran los vacíos cognitivos en el que se observa que todos los trabajos abordaban la importancia de trabajar con estrategias didácticas, pero no se evalúa las razones por las que muchas veces no se implementan estas herramientas de aprendizaje, pues una de las dificultades que se presentan en las conclusiones de las investigaciones presentadas, es que muchos maestros no usan estrategias innovadoras y los que la utilizan no lo aplican de forma adecuada.

Palabras claves: Estrategias didácticas, enseñanza, aprendizaje.

\begin{abstract}
RESUME
The purpose of this scientific article is to demonstrate the research advances on didactic strategies and their effectiveness in the teaching-learning process. In it, emphasis is placed on the methodology, corresponding to the phases of documentary research. First, the work carried out on this topic was investigated on websites, said studies are updated on a date of no less than 5 years, 50 percent national and 50 percent international. The results are also explained, which includes the theoretical contributions, that is, the conceptual foundation that explains the research variables, such as: didactic strategy and its importance in the learning process. In the same way, the cognitive gaps are shown in which it is observed that all the works addressed the importance of working with didactic strategies, but the reasons why these learning tools are often not implemented are not evaluated, as one of the difficulties that are presented in the conclusions of the research presented, is that many teachers do not use innovative strategies and those who do do not apply it adequately.
\end{abstract}

Keywords: Didactic strategies, teaching, learning.

\section{INTRODUCCIÓN}

Las estrategias de enseñanza aprendizaje son aquellas herramientas indispensables para lograr un aprendizaje con calidad, pues estas facilitan una mejor adquisición de conocimientos. Según kholer (2005) señala que las estrategias de aprendizaje «no sólo entrenan la capacidad de aprender y resolver problemas, 
sino que esto en sí mismo implica el desarrollo intelectual del estudiante, la potencialización de sus habilidades, entendiéndose éstas como estructuras flexibles y susceptibles de ser modificadas e incrementadas» (p.1). Es decir que, a través de su aplicación en las aulas de clases, el estudiantado no solo será capaz de dar respuestas a los problemas que se le presentan en su entorno, sino que este podrá desarrollar habilidades y destrezas que lo con lleven a la creatividad e innovación.

La educación es cambiante y cada día nos presenta desafíos, para ello el docente debe de irse actualizando, según la necesidad educativa, para alcanzar los objetivos que se propone la educación. Es a través de la implementación y el uso adecuado de estrategias didácticas que se puede lograr que el estudiante sea un ser protagónico de su aprendizaje, y para ello el docente debe de cambiar su forma de enseñanza y asumir nuevos retos, sin embargo, se logra apreciar que en las aulas de clases varios maestros carecen de estrategias motivadoras que ayuden a mejorar los procesos de aprendizaje. Por tal razón se decidió tratar más a fondo esta temática con el objetivo de evidenciar como las estrategias didácticas favorecen la adquisición de aprendizajes, puesto que es necesario seguir formando seres competentes para la vida.

En este estado del arte se muestran las investigaciones realizadas en cuanto a la efectividad que tienen las estrategias de aprendizaje en el aula de clase, para ello se muestran investigaciones internacionales y nacionales, en las que se valora como la aplicación de estrategias hace que las clases sean más significativas, permitiendo que el estudiantado se sienta más satisfecho y se motiven. Además, en estos estudios analizados, se resalta la importancia de trabajar con herramientas tecnológicas y actividades lúdicas, donde se cree un ambiente amigable entre docente y estudiante.

Para la construcción de este trabajo se localizaron aquellos estudios que tuvieran relación con el tema, para evidenciar de forma objetiva lo que se pretende alcanzar con esta investigación y de esta manera dar a conocer a los lectores sobre la importancia de implementar en las aulas de clase estrategias de enseñanza que favorezcan el aprendizaje significativo.

\section{METODOLOGÍA}

El estado de arte ha constituido una herramienta importante antes de llevar a cabo una investigación, porque permite conocer que se ha investigado y plantear nuevas perspectivas que no se han abordado en torno a un determinado tema. Para ello es importante emplear una metodología de selección de trabajos investigativos que hagan referencia a la temática en estudio. En este caso para esta investigación se retomaron como criterio de selección trabajos realizados tanto en el ámbito nacional como internacional, el 50 porciento de cada una, y que el tiempo máximo de publicación fuera de los últimos cinco años (2016-2021). Así mismo se tomó como punto de partida aquellos trabajos que abordaran las variables que se plantean en el título, tal como estrategias didácticas y su efectividad que tienen en el proceso de enseñanza- aprendizaje, y 
como estas influyen y facilitan la adquisición de aprendizajes significativos en el estudiantado, es decir un aprendizaje que les sirva para la vida.

La investigación fue realizada primeramente buscando en los sitios web aquellos trabajos confiables y que los resultados se presentaran de forma más cualitativa que cuantitativa, por la característica del estudio. Seguidamente dichos resultados obtenidos en la lectura de diversos trabajos se plantearon de mayor a menor en una tabla que contenía los datos bibliográficos citados según normas APA séptima edición, y se establecieron las categorías, en este caso pedagógicas y didácticas. También se analizaron los aspectos teóricos que cada uno de estos estudios presentan desde las diferentes perspectivas y contextos, ya que hay una variación en los trabajos de otros países, pues estos evidencian que en las aulas de clases se implementan varias estrategias didácticas, pero que no todas tienen la misma efectividad por la característica de cada aprendiz. A diferencia de los trabajos nacionales que más que todo se basan en la importancia de las estrategias didácticas en la práctica pedagógica.

\section{RESULTADOS}

\section{Principales conceptos.}

En cuanto a los referentes teóricos que se analizaron en las investigaciones consultadas, se percibe que las estrategias didácticas juegan un papel muy fundamental en el proceso de enseñanza-aprendizaje. Pamplona, Cuesta y Cano (2019) en su trabajo sobre estrategias de enseñanza del docente en las áreas básicas, recabó información de varias investigaciones donde se evidenciará la aplicabilidad de estrategias didácticas, obteniendo como resultado que se han realizado varias investigaciones donde se logra la identificación de diversas estrategias que faciliten la adquisición de conocimientos. Este autor menciona que estas han constituido una herramienta importante de enseñanza y para implementarlas es necesario que se realice un diagnóstico del espacio, los recursos que cuenta y las características de los estudiantes para que sean efectivas.

Las estrategias metodológicas son facilitadoras del aprendizaje, hace que este sea más accesible y dinámico, porque debe de haber una interacción entre maestro y estudiante. Todos los autores coinciden que estas son guías de acciones que hay que seguir y se deben de hacer con un objetivo, el aprendizaje funcional. Para Gutiérrez, Gómez y Ríos (2018) definen La estrategia didáctica como (...) «un procedimiento pedagógico que contribuye a lograr el aprendizaje en los alumnos, en sí, se enfoca a la orientación del aprendizaje» (p. 2). Dicho de otra manera, Ese debe de ser el norte de todo maestro parar lograr su fin, el aprendizaje significativo de los discentes. 
Jiménez e Ibarra (2015) consideran que todas las estrategias didácticas deben de estar orientadas al aprendizaje significativo en el área del desarrollo humano tanto cognitivo como emocional, en vista que se debe estimular en los estudiantes una actitud crítica y reflexiva. También plantea que los espacios lúdicos son de suma importancia para lograr el alcance de estas habilidades. Para ello se requiere que el educando muestre una actitud innovadora en los salones de clases, pues supone nuevas transformaciones para convertirse en referentes de cambio. Aunque no es una tarea fácil, es un desafío para cada una de las instituciones.

Uno de los estudios analizados muestra una nueva perspectiva de ver las estrategias didácticas, basadas en un modelo de interactividad y en un enfoque por competencias. Para el cumplimiento de este nuevo modelo el docente debe propiciar un ambiente de aprendizaje significante en los alumnos, encausar de manera interactiva un proceso de aprendizaje novedoso y activo mediante la creatividad, innovación, iniciativa, disposición y capacidad para articular los pilares fundamentales de la educación como: los saberes, habilidades cognitivas, procedimentales y actitudinales (saber, saber hacer, saber ser) (Gutiérrez, Gómez, Ríos; 2018)

Como se mencionaba con anterioridad las estrategias didácticas y su implementación en las aulas de clases se ha convertido en un desafío para muchos docentes, puesto que requiere inversión de tiempo y a veces monetarios, por tal razón muchas ocasiones no se ponen en práctica, porque el maestro se ha quedado en el conformismo y no trata de cambiar patrones de tradicionalismo a como lo menciona Molinares (2016), que varias veces los docentes sí se rigen en los programas de estudios, pero aún no se apropian de los nuevos avances que ha dado la educación, tal es el caso de las herramientas tecnológicas quienes se han convertido en una estrategia provechosa para el estudiantado y el maestro, sin embargo su aplicabilidad presenta grandes limitaciones.

Los autores sugieren que los docentes deben de cambiar de actitud y transformar su realidad en algo provechoso, pues, aunque no haya los recursos necesarios en los centros de estudios, el maestro debe de valerse de todo lo que encuentra en su entorno para dar una enseñanza con calidad. La educación requiere maestros con vocación y dispuestos al cambio, ya que el aprendizaje es procesual y asimilado de diferentes maneras.

\section{Trabajos realizados en el área}

Los estudios que se muestran en el presente estado de arte, son investigaciones realizadas sobre estrategias didácticas en el proceso de aprendizaje, tanto en el ámbito internacional como nacional, lo que permite analizar cómo se desarrolla dicha temática en ambos contextos educativos. Así mismo, cabe señalar que estos se mostrarán de mayor a menor fecha de publicación y según el lugar. 


\section{Ámbito internacional}

Fueron pocos los trabajos monográficos encontrados en relación a la temática en estudio, sin embargo, se localizaron revistas que hacen énfasis al tema de investigación. Pamplona (2019) desarrolla en Colombia, el tema de las estrategias de enseñanza del docente en las áreas básicas: una mirada al aprendizaje escolar, esta tenía como finalidad identificar las estrategias pedagógicas que se implementan con mayor frecuencia en la escuela, tradicionales e innovadoras, a través de las diversas investigaciones que se han realizado en cuanto a estrategias de aprendizaje.

Para la realización de esta se recurrió al enfoque cualitativo, a partir del método de investigación documental de tipo descriptivo no experimental, puesto que se hizo una revisión de la literatura que se ha trabajado en torno a la importancia de las estrategias didácticas en el proceso educativo. En las delimitaciones muéstrales se priorizaron las investigaciones realizadas entre el 2011 y 2017 que tuvieran como propósito resaltar la importancia de las estrategias de enseñanza en las áreas básicas de primaria; y posteriormente hacer el análisis por medio de una ficha analítica, donde se registraron los resultados obtenidos.

La conclusión a la que llegó Pamplona durante su estudio, es que, según las investigaciones analizadas, todavía hay maestros que siguen enseñando con patrones tradicionalistas, no obstante, se evidencia que varios docentes han tratado de innovar, haciendo usos del juego, las TIC, el arte y dinámicas grupales, favoreciendo de esta manera el aprendizaje integral de los educandos. Por tanto, es necesario que estos maestros divulguen la práctica pedagógica para que aquellos educandos que aún siguen estancados en el tradicionalismo puedan conocer nuevas estrategias didácticas que faciliten el aprendizaje. Es muy pertinente el estudio realizado por este autor para este trabajo, en vista que permite reflexionar desde su contexto como las estrategias didácticas vienen a enriquecer el trabajo educativo y cuán importante es cambiar a diario nuestra forma de enseñanza, así mismo permite analizar que a nivel de países encontramos maestros con la misma actitud.

El proceso de enseñanza no es estático, día a día el profesorado debe de apropiarse de estrategias de aprendizaje que faciliten la adquisición de un aprendizaje significativo del estudiantado. Gutiérrez, J. et al (2018) llevan a cabo el estudio "estrategias didácticas de enseñanza y aprendizaje desde una perspectiva interactiva", esta tiene por objetivo demostrar como las estrategias metodológicas de enseñanza, aprendizaje y evaluación con un enfoque interactivo, son recursos didácticos que coadyuvan a lograr que la enseñanza se convierta en una acción lúdica, es decir, una enseñanza basada en juegos.

El estudio de estos autores se desarrolló por medio de una investigación acción (trabajo basado en una propuesta). La propuesta consiste en aplicar estrategias didácticas lúdicas interactivas desde la práctica docente. La muestra se basó en 7 grupos de licenciatura: 6 de la Licenciatura en Educación Secundaria de la Especialidad en Matemáticas (120 alumnos) y 1 en la Especialidad de Español (24 estudiantes). También se estructuró 
un programa académico de capacitación y actualización a maestros en servicio de los diferentes niveles educativos del estado de México, considerando los contextos socioculturales rural, semiurbano y urbano, con la intención de tener un bosquejo amplio sobre la importancia de impulsar una enseñanza interactiva dentro de las aulas de clase.

A partir del estudio realizado los autores obtienen como resultado que «la propuesta académica de enseñanza con un enfoque lúdico e interactivo, conlleva a reflexionar sobre la trascendencia que tiene el documentar las experiencias innovadoras de enseñanza y conduce al sujeto investigador a sistematizar y documentar los hechos cotidianos» (Pamplona. et al. 2018. p.12). Es decir que consideran importante el enseñar a través del juego, objetivo principal del modelo educativo de Nicaragua, que los estudiantes aprendan jugando, para despertar el interés y la creatividad en el estudiantado. Por tanto, este estudio presentado viene a fortalecer y a reafirmar la preocupación que tiene nuestra educación al alinearse a nuevas formas de enseñanza, donde el estudiante pueda sentirse en confianza con el aprendizaje.

Jiménez e Ibarra en el año 2015 realizan la investigación Implementación de estrategias didácticas orientadas al aprendizaje significativo en el área de desarrollo humano. Este desde un sentido más amplio reflexiona como las estrategias didácticas en el proceso de enseñanza deben de estar encaminadas al aprendizaje integral de los estudiantes, donde no solamente se valore conocimientos científicos, si no también valores, habilidades, destrezas, capacidades, etc. Por consiguiente, el maestro debe de estar interesados en utilizar estrategias que propicien el trabajo activo de los estudiantes y su desarrollo intelectual.

Se considera necesario la conclusión a la que llegan estos autores, porque analizaron las estrategias didácticas que el maestro puede utilizar para lograr el objetivo de la educación. Así mismo que importante es adoptar una metodología y el modelo de evaluación para la implementación de estrategias didácticas que facilite la educación, que sean herramientas versátiles en cada situación educativa particular y de necesidad individual. Una vez más se logra apreciar como nuestro objetivo de educación no está alejado de contextos educativos internacionales y que es importante que se continúe la actualización de los maestros en cuanto a herramientas de enseñanza para lograr la calidad educativa.

\section{Ámbito nacional}

En el contexto nacional se localizan 2 trabajos que más se asimilan al tema en estudio. Uno de ellos corresponde a Molinares (2016) con el tema Incidencia de la aplicación de estrategias metodológicas de la docencia en el proceso de aprendizaje de estudiantes de IV año, carrera de ciencias sociales, facultad regional multidisciplinaria de Matagalpa I/ semestre, 2014. En el estudio se analizaron las incidencias que tienen las estrategias didácticas en la interacción entre docentes y estudiantes, pues estas facilitan pensamiento autó- 
nomo, la criticidad, creatividad, la capacidad innovadora, esto con la intención clara de aportar soluciones a los distintos problemas de la vida social, que es lo que se conoce como aprendizaje significativo.

El estudio se basó en un enfoque cuantitativo para mostrar numéricamente los datos y cualitativo para describir los datos numéricos. Se tomó una población de 32 estudiantes de cuarto año de la carrera de Ciencias Sociales, más 10 docentes de la Facultad Regional Multidisciplinaria de Matagalpa; todos fueron parte de la muestra, ya que consideran que era una cantidad pequeña. Para la recolección de la información se aplicaron entrevistas a los docentes y una encuesta a los estudiantes de dicha carrera, así mismo se observó el desarrollo de una clase para obtener un mejor resultado.

Los principales resultados encontrados es que la mayoría de maestros tienen dominio científico y pedagógico al impartir su clase; el estudiante se siente a gusto cuando el maestro realiza actividades creativas para impartir su clase. Por tanto concluyen con este estudio que el éxito del proceso de aprendizaje en estudiantes está asociado al dominio científico técnico del maestro, de igual manera los docentes consideran que es productivo que los estudiantes también demuestren disposición al momento del desarrollo de la clase, pues esto facilita que haya un ambiente de interacción entre docente y discente. Este estudio refleja como en la educación superior los docentes valoran la importancia de continuar trabajando con estrategias didácticas creativas y que faciliten el aprendizaje oportuno de los jóvenes que se continúan preparando.

El segundo pertenece a Fuentes (2015) quien realiza el trabajo Efectividad de las estrategias didácticas que implementan los docentes en la formación de estudiantes, curso de profesionalización, esta investigación se realizó en la UNAN-Farem Estelí y pretendía analizar la relevancia del roll mediador que desempeña el docente al acompañar a los estudiantes en su formación profesional, en vista que es fundamental el papel que juega I docente el quehacer educativo, pues el estudiante lo ve como un modelo a seguir.

Dicha investigación se desarrolló bajo un enfoque cualitativo, porque se describieron los hallazgos encontrados. Para la recolección de la información se hizo uso de entrevistas, observaciones, grupo focal y análisis documental con una muestra pequeña de estudiantes quienes tenían como tarea valorar como sienten la adquisición de aprendizaje cuando un maestro hace uso estrategias didácticas motivadoras y cuando no las utiliza. Los instrumentos aplicados arrojaron que todos los docentes que participaron en el estudio aplican estrategias didácticas para el desarrollo del proceso de aprendizaje, sin embargo, algunas de estas estrategias son repetitivas y monótonas, lo que de alguna imposibilita el aprendizaje significativo de los estudiantes.

Los estudiantes encuestados afirman que sienten más accesible el aprendizaje cuando sus maestros aplican estrategias didácticas. A través de este estudio se observa de cerca como en nuestro país aún hay 
maestros que continúan estancados en el tradicionalismo y se las hace difícil enseñar a través de actividades motivadoras que con lleven al estudiante a construir su propio aprendizaje.

\section{Vacíos cognitivos}

En las investigaciones realizadas sobre estrategias didácticas y su importancia en los diferentes niveles educativo, se encuentra que todos los estudios mencionan la importancia de como las estrategias de aprendizaje, propician un aprendizaje significativo, sin embargo, no se muestra por qué algunos maestros, a pesar de que existen un sinnúmero de estrategias metodológicas no la usan en los salones de clase, lo que significa que se ha convertido en un desafío en los diferentes contextos educativos, lograr la aplicación de estrategias metodológicas. También se percibe que en las soluciones no se brindan medidas de cómo lograr que los docentes tomen conciencia sobre el roll que tienen dentro de los salones de clase y que es importante educar seres competentes para dar solución a cualquier situación que se le presente en su entorno.

Cabe mencionar que dentro de estas investigaciones vale analizar y mostrar resultados de cómo influyen de manera significativa el uso de estrategias didácticas, para que los estudiantes desarrollen habilidades del pensamiento crítico, es decir, no solamente mostrar aspectos teóricos de cómo dichas formas de enseñanza son importante, sino más bien demostrar porque tenemos dificultades en que el maestro se apropie de actividades creativas. De la misma manera se considera necesario presentar un análisis de los aprendizajes de los estudiantes en donde el maestro le enseña por medio metodologías de enseñanza innovadores, como las que mencionaba pamplona (2019) en el que afirma que el estudiantado aprende a través de actividades lúdicas, porque se requiere que se trabaje bajo una educación integral.

\section{CONCLUSIONES}

El sistema educativo de Nicaragua está centrado en el aprendizaje integral de los estudiantes, que sean competentes para dar solución a las diferentes problemáticas que se le puedan presentar en su entorno. Para ello se requiere que el docente continúe su formación científica pedagógica, para el logro de esto el Ministerio de Educación local ha implementado diferentes medios o recursos para que los docentes se preparen; entre ellos están: los cursos cortos, encuentros de actualización permanente, cursos de formación continua, entre otras; estas con el fin de que el docente se apropie de estrategias didácticas que coadyuven el proceso de enseñanza-aprendizaje.

A pesar que se está trabajando para lograr la calidad educativa con diferentes cursos de actualización, ha sido un reto lograr que algunos docentes apliquen diferentes estrategias para desarrollar contenidos y lograr un aprendizaje significativo, puesto que aun encontramos maestros con modelos educativos tradiciona- 
listas y esto de una u otra manera ha venido a dificultar que los estudiantes se motiven y se interesen por las clases. Por esa razón es importante analizar lo que se ha investigado sobre el uso de estrategias de enseñanza en aula de clase en los diferentes contextos y conocer sobre la importancia que tienen dichas estrategias en el quehacer educativo.

\section{REFERENCIAS}

Fuentes, E. (2016). Efectividad de las estrategias didácticas que implementan los docentes en la formación de estudiantes, curso de profesionalización. Revista tesis. https://repositorio.unan.edu.ni/3104/

Gutiérrez, J., Gómez, F. y Gutiérrez, C. (2018). Estrategias didácticas de enseñanza y aprendizaje desde una perspectiva interactiva, 1-15. http://www.conisen.mx/memorias2018/memorias/2/P845.pdf

Jiménez, M. e Ibarra, H. (2015) Implementación de estrategias didácticas, orientadas al aprendizaje significativo en el área de desarrollo humano. Revista Ecofarn, 53-61. https://www.ecorfan.org/proceedings/ CDU_IV/CDUIV_7.pdf

Kohler Herrera, J. (2005). Importancia de las estrategias de enseñanza y el plan curricular. Liberabit, 11(11), 25-34. http://pepsic.bvsalud.org/scielo.php? script=sci_arttext\&pid=S172948272005000100004\&lng=pt\&tlng=es.

Molinares Centeno, J.R. (2016). Incidencia de la aplicación de estrategias metodológicos de la docencia en el proceso de aprendizaje de estudiantes de IV año, carrera de ciencias sociales, facultad regional multidisciplinaria de Matagalpa, /l semestre 2014 [tesis de maestría, universidad Nacional Autónoma de Nicaragua]. https://repositorio.unan.edu.ni/1867/1/5342.pdf

Pamplana, J., Cuesta, J. y Cano, V. (2019). Estrategias de enseñanza del docente en las áreas básicas: una mirada al aprendizaje escolar. Revista Eleuthera, (219, 14-33. http://www.scielo.org.co/pdf/eleut/ v21/2011-4532-eleut-21-00013.pdf 